\title{
Preservation of Hearing in Cochlear Implant Surgery: Advantages of Combined Electrical and Acoustical Speech Processing
}

\author{
Bruce J. Gantz, MD; Christopher Turner, PhD; Kate E. Gfeller, PhD; Mary W. Lowder, MA
}

\begin{abstract}
Objectives/Hypothesis: This study documents the importance of preserving residual low-frequency acoustic hearing as those with more residual hearing are selected for cochlear implantation. Surgical strategies used for hearing preservation with a short hybrid cochlear implant are outlined. The benefits of preserved residual low-frequency hearing, improved word understanding in noise, and music appreciation are described. Study Design: Multicenter, prospective, single-subject design. Methods: Records were reviewed of 21 individuals participating in an Food and Drug Administration (FDA) feasibility clinical trial who have received an Iowa/Nucleus $10 \mathrm{~mm}$ electrode. A second group of subjects receiving implants at the University of Iowa that have used the $10 \mathrm{~mm}$ device between 2 years and 6 months were also reviewed. Outcome measures included standardized tests of monosyllabic word understanding, spondees in noise, and common melody recognition. Results: Lowfrequency hearing was maintained in all individuals immediately postoperative. One subject lost hearing at 2.5 months postoperative after a viral infection. The group has averaged a loss of $-9 \mathrm{~dB}$ low-frequency acoustic hearing between 125 and 1,000 Hz. Monosyllabic word understanding scores at 6 months for a group being followed for an FDA clinical trial using the implant plus hearing aids was $69 \%$ correct. For the long-term group receiving implants at Iowa,
\end{abstract}

From the Department of Otolaryngology_-Head and Neck Surgery (B.J.G., C.T., M.W.L.), University of Iowa Roy J and Lucille A Carver College of Medicine; the Department of Speech Pathology and Audiology (C.T., K.E.G.); and the School of Music (K.E.G.), The University of Iowa, Iowa City, Iowa, U.S.A.

Supported (in part) by research grants 2 P50 DC00242 and 1 R01 DC 000377 from the National Institutes on Deafness and Other Communication Disorders, National Institutes of Health; grant RR00059 from the General Clinical Research Centers Program, NCRR, National Institutes of Health; the Iowa Lions Sight and Hearing Foundation; and Cochlear Corporation for developing a cochlear implant to our specifications, providing initial devices at no cost, and sharing data from their ongoing clinical trial with the Hybrid implant. $19,2005$.

Editor's Note: This Manuscript was accepted for publication January

Send Correspondence to Dr. Bruce J Gantz, Department of Otolaryngology-Head and Neck Surgery, University of Iowa Hospitals and Clinics, 200 Hawkins Drive (21201 PFP), Iowa City, IA 52242-1078, U.S.A. E-mail: bruce-gantz@uiowa.edu

DOI: 10.1097/01.MLG.0000157695.07536.D2 monosyllabic word understanding in those who have used the device between 6 months and 2 years is $79 \%$. Other important findings include improved recognition of speech in noise (9 dB improvement) as compared with standard cochlear implant recipients who were matched for speech recognition in quiet and near normal recognition of common melodies. Conclusion: The surgical strategies outlined have been successful in preservation of low-frequency hearing in 96\% of individuals. Combined electrical and acoustical speech processing has enabled this group of volunteers to gain improved word understanding as compared with their preoperative hearing with bilateral hearing aids and a group of individuals receiving a standard cochlear implant with similar experience with their device. The improvement of speech in noise and melody recognition is attributed to the ability to distinguish fine pitch differences as the result of preserved residual low-frequency acoustic hearing. Preservation of low-frequency acoustic hearing is important for improving speech in noise and music appreciation for the hearing impaired, both of which are important in real-life situations. Key Words: Hearing Preservation, cochlear implant, hybrid cochlear implant, hearing in noise.

Laryngoscope, 115:796-802, 2005

\section{INTRODUCTION}

The cochlear implant (CI) has been able to restore substantial hearing in profound prelingually deafened children and profound postlingually deafened adults and children. The remarkable word understanding scores obtained by these groups of individuals has enabled those with less than profound deafness to be considered for implantation. Expansion of the selection criteria for implantation to those with more residual hearing is supported by data that residual hearing is one of the few variables identified that directly impacts speech perception results. ${ }^{1}$

In this article, we describe a somewhat different rationale for considering the patients' residual hearing in cochlear implantation. The decision to undergo traditional implantation surgery will usually mean that patients' residual acoustic hearing is usually no longer available. However, the residual low-frequency acoustic hearing, 
when it can be used by the patient in addition to electrical stimulation, has some important advantages compared with traditional "electric only" CIs. Therefore, there are a number of disadvantages that should be considered before the decision to destroy residual acoustic hearing is undertaken. The electrical signal-processing algorithm and stimulation of the auditory nerve that provides important information for speech perception in quiet is reported by users of CIs as "mechanical" or "raspy" when compared with their memories of acoustic hearing. In addition, the loss of pitch perception, which is primarily a consequence of the limited spectral resolution of current CI speech processing, has unfortunate consequences for the perception of speech in noise and for the accurate perception and enjoyment of music.

Although poor pitch resolution does not appear to be a limitation for understanding speech in quiet for the most successful implant users, ${ }^{2}$ understanding speech in background noise requires spectral resolution even finer than that required for understanding speech in quiet. ${ }^{3}$ Even the most successful implant users only realize perhaps 6 to 8 channels of distinct "place-frequency" information across the entire spectral range, and this deficit in spectral resolution has a direct negative consequence on the implant user's ability to understand speech in background noise. ${ }^{4}$ Thus, although traditional CIs are quite successful for many, if not most, patients in restoring excellent speech recognition in quiet, even the most successful implant patients suffer from significant problems understanding speech in background noise. ${ }^{5,6}$ Previous results from our clinic have shown that preservation of residual low-frequency acoustic hearing can improve speech recognition in noise for implant patients. ${ }^{5}$

With regard to music, implant recipients using conventional long electrode implants have shown poorer performance than normal hearing persons on several pitchrelated tasks, including detecting pitch change (frequency difference limens), perception of direction of pitch change (higher or lower), and discrimination of brief pitch patterns. ${ }^{7,8}$ CI recipients using conventional long electrodes almost always require considerably larger frequency differences than normal-hearing adults for detecting pitch change (frequency difference limens) as well as the direction of a pitch change (i.e., whether the second pitch of a pair of notes is higher or lower than the first). ${ }^{7}$

Poor pitch resolution has a detrimental effect on the esthetic quality of musical sounds, and it also impedes the CI user's ability to perform a very basic listening task that normal hearing people tend to take for granted: the ability to recognize familiar melodies such as "Happy Birthday," "The Star Spangled Banner," holiday songs, and spiritually-significant songs when no lyrics are provided. Melody recognition requires the listener to hear a sequence of pitches and accurately perceive the direction of pitch change (higher or lower) as well as the magnitude of each pitch change. Because melodies are made up of sequential pitch patterns, poor perception of pitch, as is common for CI recipients who use the conventional long electrode, has negative implications for this perceptual task. ${ }^{7,9}$ In short, because acoustic hearing has an important role in pitch resolution and those hearing tasks facil- itated by pitch, the loss of residual hearing is an important clinical consideration when determining the most appropriate options for patients with severe hearing losses.

The loss of residual acoustic hearing during implantation is the result of a combination of factors including the technique used to create the cochleostomy as well as the size of cochleostomy performed. In addition, the diameter, stiffness, and length of standard intracochlear electrodes may induce substantial intracochlear damage to the basilar membrane and cochlear hair cells as they advance around the upper basilar turn during insertion. This damage is likely caused by forces against the outer cochlear wall during the insertion (Tom Roland, personal communication, submitted for publication).

In an attempt to limit the intracochlear damage and to preserve residual low-frequency hearing in individuals with poor word understanding (less than 50\% CNC word understanding), a short $10 \times 0.4 \times 0.2 \mathrm{~mm}$ electrode (Iowa/Nucleus Hybrid Cochlear Implant) has been developed. ${ }^{10,11}$ Volunteers receiving an implant with this device have usable acoustic hearing up to 1,000 Hz. Electrical speech processing stimulation provides the patient with high-frequency speech information (usually 1,500$6,000 \mathrm{~Hz}$ ). Thus, these subjects perceive sound by way of a "combined acoustic and electric" $(\mathrm{A}+\mathrm{E})$ mode. They report that the esthetic qualities of sound are preserved, that they enjoy listening to music, and that they exhibit better speech recognition in background noise. ${ }^{5}$

This report describes the unique aspects of the technique that have been used for implantation of the Iowa/ Nucleus Hybrid Implant. In addition, we discuss longterm results, preliminary results of an ongoing Food and Drug Administration (FDA) trial to support the concept that low-frequency hearing can be preserved, and expand on the rational for preservation of residual hearing.

\section{MATERIALS AND METHODS}

\section{Surgical Technique}

The Iowa/Nucleus Hybrid Implant was designed to limit damage to the scala media. The intracochlear electrode has a reduced diameter of $0.2 \times 0.4 \mathrm{~mm}$. Unique features of the electrode include a Dacron collar to limit the intracochlear placement to $10 \mathrm{~mm}$ and a titanium marker to orient the electrode contacts, thus ensuring the correct alignment of the electrodes adjacent to the modiolus. The length of the electrode was limited to $10 \mathrm{~mm}$ to prevent injury to the ascending basal turn of the cochlea and prevent the electrode from curling on itself on insertion.

A mastoidectomy, the creation of the bony seat for the electronic package, and the facial recess is opened similarly to implantation of a standard CI. The bony overhang of the round window niche is removed with a $1.8 \mathrm{~mm}$ diamond burr to expose the round window membrane.

The strategy for creating the cochleostomy and entering the scala tympani should involve surgical detail similar to performing a "drill-out" stapes for otosclerosis. Bleeding must be controlled, and suction must be avoided once the inner ear is opened. The cochleostomy is created anterior inferiorly to the round window. To standardize the placement of the cochleostomy, it is suggested that the cochleostomy is placed in the anterior inferior quadrant of a box created by drawing a line at the superior margin of the round window and one that crosses perpendicular at the inferior aspect of the round window (Fig. 1). 


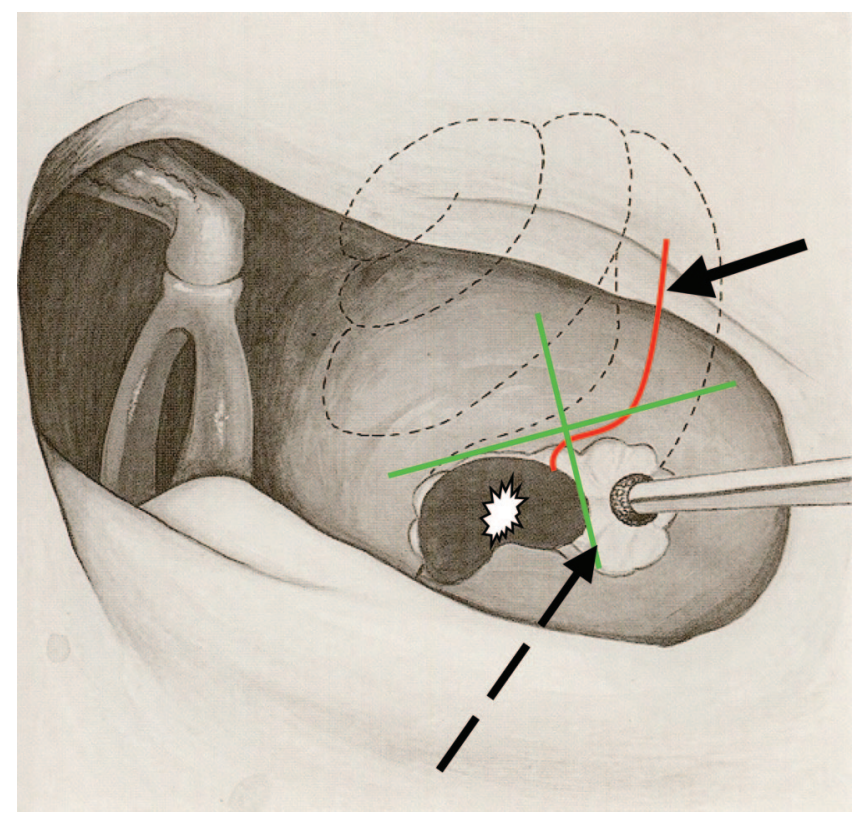

Fig. 1. Position of cochleostomy in the inferior-posterior quadrant of a box created by drawing a line at the superior margin of the round window and a perpendicular line at the inferior margin of the round window (dashed arrow). Basilar membrane position (solid arrow), round window membrane (star).

Creation of the cochleostomy is begun in the inferior portion of the quadrant, slowly saucerizing the otic capsule bone with a 1 $\mathrm{mm}$ diamond bur. Approaching the scala tympani from a caudal direction ensures that the cochleostomy will be inferior to the equator of the scala, thus avoiding the basilar membrane and spiral ligament (Fig. 2). It should be remembered that the basal turn of the cochlea is oriented obliquely in the temporal bone, with the helocotrema usually positioned deep to the tensor tympani muscle, and the ascending basilar turn can be deep to the level of the internal carotid artery. Because bone is slowly removed over the scala tympani, the lumen will appear as bluish or as a faint gray hue. If the cochleostomy is too far superior, adjacent to the spiral ligament, a whitish color will be evident. Drilling inferiorly will permit entrance into the scala tympani.

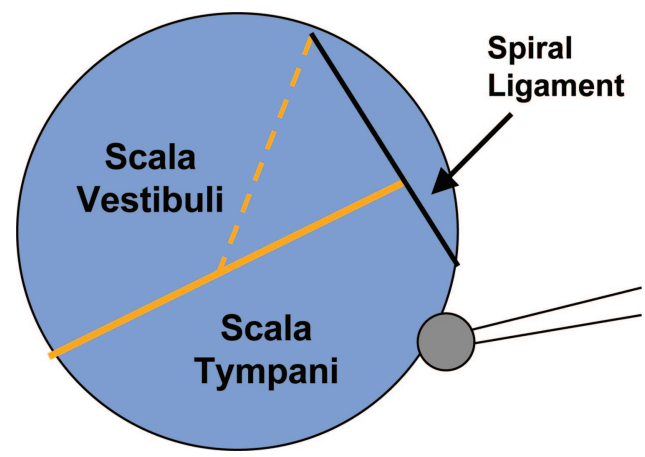

Fig. 2. The cochlea is oriented in the temporal bone such that the basal turn is tipped anterior-superior with the helocotrema deep to the tensor tympani muscle. The black vertical line indicates the approximate position of the spiral ligament. The cochleostomy should be placed inferior to the spiral ligament to prevent damage to the basilar membrane.
A $0.5 \mathrm{~mm}$ diamond bur is used to penetrate the final layer of bone, but the endosteum of the scala tympani is left intact (Fig. 3 ). The implant is secured in the seat and sutured in place with 4-0 nylon suture. During creation of the mastoid, an overhang of the cortex at the tegmen mastoideum is developed. One millimeter holes are drilled in the cortical overhang to secure the electrode with a 4-0 nylon suture before it is placed in the cochlea. This suture helps to stabilize the orientation of the electrode contacts toward the modiolus and ensures that the electrode is secure within the scala tympani. Before placement of the electrode, $1.5 \times 1.5 \mathrm{~mm}$ of temporalis fascia is obtained and pressed in a fascia press. A needle is used to pierce the fascia in the center. The electrode tip is threaded through the fascia, creating a washer that is advanced against the Dacron cuff. The fascia seals the cochleostomy when the electrode is inserted into the scala tympani. The endosteum is opened using a $0.2 \mathrm{~mm}$ rightangle hook. The tip of the electrode is guided into the cochleostomy using a needle tipped instrument at the Dacron collar and an electrode-guiding "claw" instrument. The electrode must be directed parallel to the posterior canal wall to ensure the electrode is directed into the basal turn of the cochlea. The electrode is then slowly advanced into the scala tympani. A slow insertion (over a 30-45 second period) will limit the intracochlear trauma and allow displacement of perilymph. The electrode is positioned with slight anterior pressure to ensure the electrode is held tightly in the cochlea, and the tegmen mastoideum suture is secured. The subperiosteal tissues and skin are closed in layers.

\section{Subjects}

Thirteen volunteers participated in this research at the University of Iowa. The first three Iowa subjects received a $6 \mathrm{~mm}$ electrode. Their data are used to support the preservation of residual hearing only. Eleven additional subjects received implants at participating research centers by seven different surgeons. The $10 \mathrm{~mm}$ electrode subjects $(\mathrm{n}=21)$ are enrolled in an FDA multicenter clinical trial. This investigation is controlled under an FDA feasibility IDE \#G990155 and has been approved by the University of Iowa Human Subjects Investigational Review Board. The criteria for selection include postlingual hearing impairment CNC word understanding between $10 \%$ and $50 \%$ with an appropriately fitted hearing aid in the worse ear, and $60 \%$ or worse in the better hearing ear. The criteria for inclusion

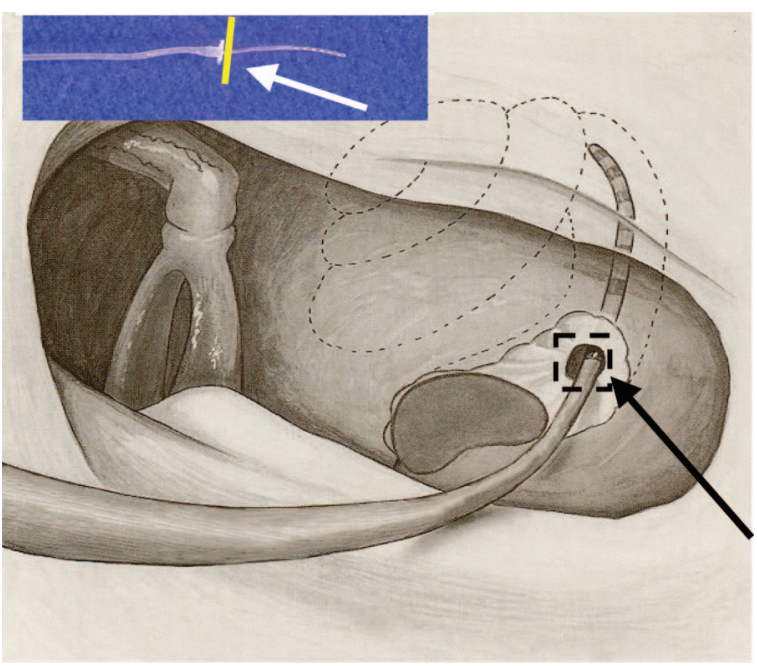

Fig. 3. The electrode is passed through a fascia washer and is placed against the Dacron collar (white arrow). The fascia washer seals the cochleostomy site (dotted line box, black arrow) 
also includes pure tone audiogram thresholds conforming to the limits shown in Figure 4.

The original $6 \mathrm{~mm}$ device produced an unpleasant highpitch percept. The electrode was lengthened to $10 \mathrm{~mm}$ to place the electrode array at a more apical location, with the most apical electrode now at approximately 2,500 to $3,000 \mathrm{~Hz}$ according to the Greenwood place frequency map. ${ }^{12}$ The tip of the $10 \mathrm{~mm}$ electrode curves into the ascending segment but does not extend to the upper basal turn of the cochlea. The added electrode length has eliminated the high-pitched percept.

Subjects were tested in a sound field with their implant alone, hearing aid + implant (hybrid condition), and hearings aids binaurally + implant (combined condition). A recorded version of the CNC word recognition test was administered to the Iowa and participating center subjects. ${ }^{13}$ The $10 \mathrm{~mm}$ Iowa Hybrid recipients were also tested using a closed-set spondee word list presented in multitalker background noise. ${ }^{5}$ The music perception task demonstrates the ability to recognize open set melodies well known within American culture presented in a pitch range of 131 to $1,048 \mathrm{~Hz}$. A description of this test and the outcomes for normal-hearing adults and CI recipients of conventional long electrodes appear in Gfeller et al. ${ }^{7}$

\section{RESULTS}

The method of performing the cochleostomy and insertion of a 6 or $10 \mathrm{~mm}$ electrode described above has enabled the preservation of acoustic hearing at 1 month postoperatively in all 24 volunteers undergoing the procedure by multiple surgeons. One subject developed a recurrent cytomegalovirus infection 2.5 months postoperative, resulting in total loss of acoustic hearing in the implant ear. Some acoustic hearing has returned at 6 months. A second patient at a participating center experienced a 30 $\mathrm{dB}$ pure hearing drop after an episode of pneumonia 3 months postoperatively. A pure tone drop of $9.5 \mathrm{~dB}$ across the frequencies between 125 and $1,000 \mathrm{~Hz}$ was noted for the group presently being tracked for an FDA investigation (Fig. 5). The range of change was $0 \mathrm{~dB}$ to $30 \mathrm{~dB}$.

Preliminary results from those reaching the 6 month experience interval in the FDA study $(\mathrm{n}=11)$ are shown in Figure 6. Monosyllabic word understanding at 3 months and 6 months compared with the preoperative

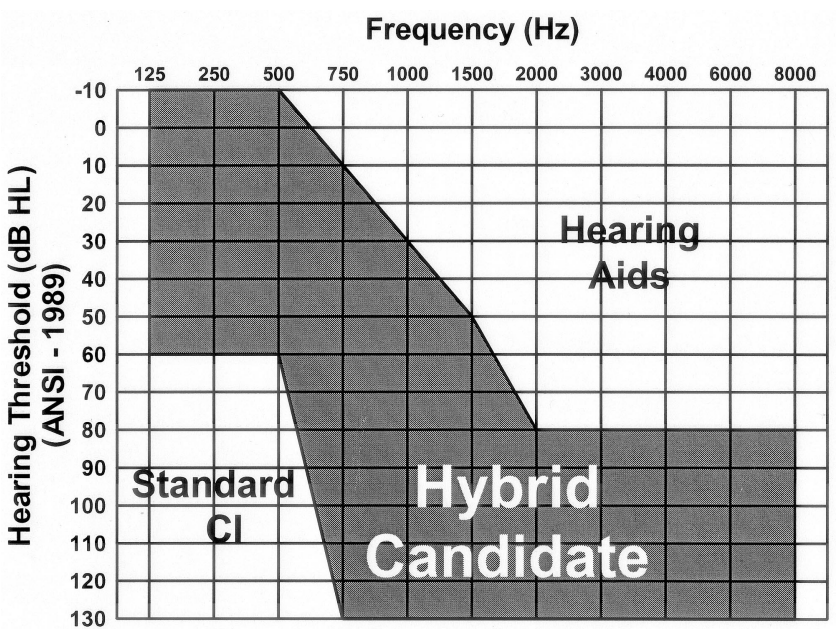

Fig. 4. Audiometric profile (gray area) for lowa/Nucleus Hybrid Food and Drug Administration trial. binaural hearing aid condition demonstrate substantial improvement in 10 of the 11 subjects. Longer-term monosyllabic word understanding scores for the subjects receiving implants at Iowa using the implant only, implant plus hearing aid, and implant plus binaural hearing aids compared with the preimplant scores with hearing aids are shown in Figure 7. The average score for this group is $79 \%$ in the best-aided condition. Some do better without a hearing aid in the implanted ear because they believe that the hearing aid blocks residual low-frequency hearing. It appears that the gains in word understanding in those that have had their devices for up to 3 years remains.

An important finding when acoustic and electrical stimulation is combined was the improved performance for recognizing speech in a noisy background as compared with traditional CI patients. The background was a twotalker babble signal. Eight of the Iowa patients receiving implants with the $10 \mathrm{~mm}$ electrode have been tested to determine the signal-to-noise $(\mathrm{S} / \mathrm{N})$ ratio required for $50 \%$ correct recognition of spondees. For comparison purposes, the same measure was obtained for a group of 20 traditional long-electrode patients (who were chosen so that their group speech recognition scores in quiet matched that of the $8 \mathrm{~A}+\mathrm{E}$ patients) as well as 10 mild-to-moderate hearing loss (HL) patients (some who wore hearing aids) and also a group of 8 normal-hearing subjects. A lower S/N ratio indicates better performance. The babble background is a situation that commonly yields complaints from all hearing-impaired patients, and the data of Figure 8 indicate that normal-hearing listeners can do quite well in this situation. It is easily seen that the $\mathrm{A}+\mathrm{E}$ approach yields an improvement over the traditional implant patients (who perform the most poorly of all groups) and that the preservation of residual hearing can help implant patients using $\mathrm{A}+\mathrm{E}$ to nearly equal the level of performance of mild-to-moderate HL patients.

Standard CI recipients report difficulty recognizing music and familiar melodies. ${ }^{7}$ This group of shortelectrode implant users is substantially more accurate than recipients of standard CIs in melody recognition, pure tone frequency discrimination, and timbre ratings for the low frequencies.

In the open-set test of familiar melody recognition, normal hearing adults $(n=17)$ achieved a mean score of $87.1 \%$ correct, whereas the mean score for 27 recipients of long electrodes (Nucleus 22, Nucleus 24, and Clarion users; ACE, Speak, CIS, and SAS speech processing strategies) was $30.7 \%$ correct. There were no significant differences among the long-electrode recipients as a function of either device or strategy. In contrast, the mean score for familiar melody recognition for five short-electrode recipients 1 year post hook-up was $80.1 \%$ correct. In summary, the hybrid short electrode subjects were significantly more accurate than traditional long-electrode implant recipients in the pitch-based task of familiar melody recognition. The A + E strategy, where residual acoustic hearing up to 750 to $1,000 \mathrm{~Hz}$ is preserved, allows the fundamental frequencies of many melodies to be perceived by way of the residual acoustic hearing, where significant advantages in pitch perception exist as compared with electric hearing. 


\section{Mean Unaided Audiometric Data US Multicenter Subjects ( $N=21)$}

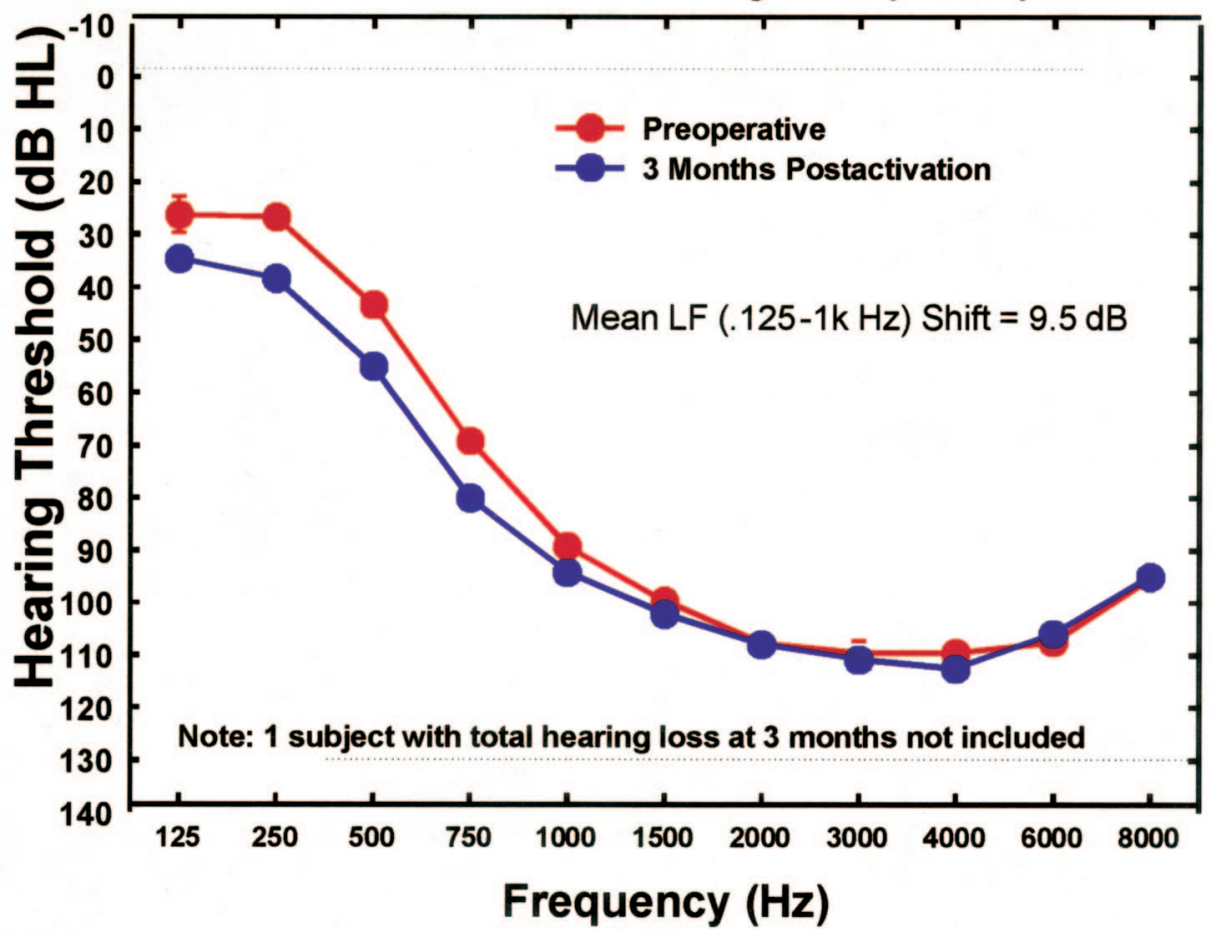

Fig. 5. Mean unaided pure tone audiometric results for 21 subjects receiving implants with the $10 \mathrm{~mm}$ electrode.

\section{DISCUSSION}

Preservation of residual low-frequency hearing with the addition of electrical speech processing has substantially improved the speech perception abilities and hearing in noise of this group of volunteers. Music appreciation and quality of sound have also been maintained. This is not a trivial consideration given the ubiquitous nature of music in our society as well as the fact that implant recipients report enjoyment of music as their most important wish after improved speech perception. ${ }^{7}$ The importance of preserving residual low-frequency acoustic hearing cannot be underestimated in view of the observation that the added benefit of hearing in noise and quality of music is a result of the residual acoustic hearing in this population. This residual hearing provides finer spectral resolution than what is possible from the present CI 10mm Subjects CNC Word Recognition:

\section{3 and 6 mo}

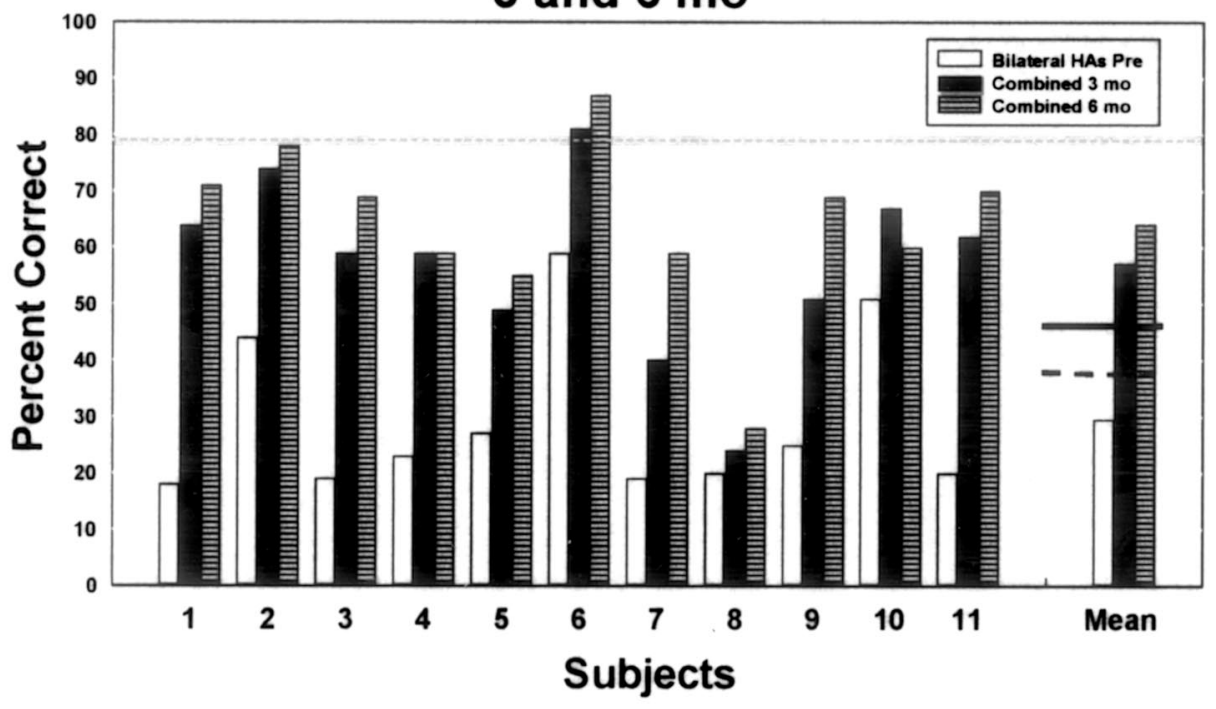

Fig. 6. Ten millimeter subjects in a Food and Drug Administration feasibility trial 3 months (black bar) and 6 months (dashed bar) compared with their preoperative hearing with two hearing aids (white bar). Mean scores are found at right. Average performance for a group of standard length electrode subjects with the traditional Nucleus Cl-24 implant are represented by the dashed line ( 3 months) and solid line (6 months). 


\section{CNC Word Scores $10 \mathrm{~mm}$ Electrode Subjects lowa Subjects}

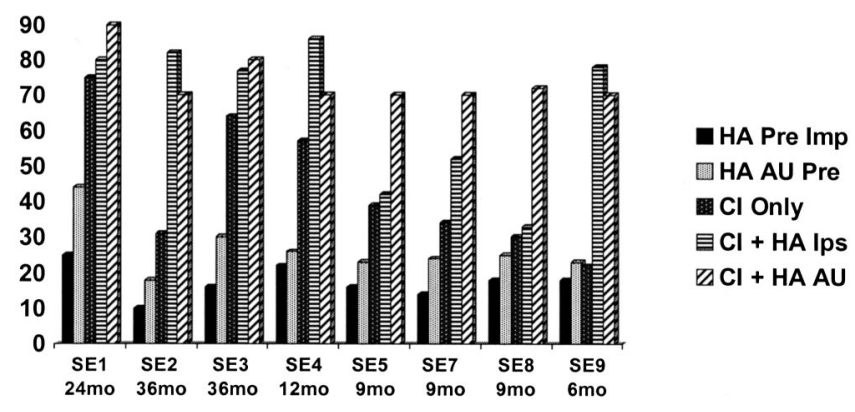

Fig. 7. Long-term results on monosyllabic CNC word score test for lowa subjects with $10 \mathrm{~mm}$ electrodes. Preoperative hearing in the implant ear (solid black bar), preoperative performance with two hearing aids (stippled bar), performance in the implant only condition (black with white dotted bar), hearing aid plus implant (horizontal bar), hearing aids binaural plus implant performance (slanted bar).

speech-processing algorithms. The $10 \mathrm{~mm}$ electrode has been successful in preserving hearing and providing sufficient additional high-frequency information to improve speech perception. A balance between intracochlear trauma, acoustic hearing, and delivery of electrical current has been achieved. It could be argued that a longer electrode may deliver more information; however, if individuals with significant residual hearing are to benefit from this technology, preservation of residual hearing is of paramount consideration. It is interesting that some subjects achieve between $60 \%$ and $70 \% \mathrm{CNC}$ word recognition with a six channel $10 \mathrm{~mm}$ electrode using electrical speech processing only without acoustic hearing. The risk/benefit performance ratio of combined electrical and acoustical stimulation is just beginning to be explored. Another concern is the potential deterioration of residual lowfrequency hearing over time. In a review of our clinic's records for adults with severe high-frequency HL, lowfrequency (less than 2,000 Hz) thresholds remain remarkably constant over many years (up to 20 years) and lose less than $0.75 \mathrm{~dB} /$ year on average.

$\mathrm{A}+\mathrm{E}$ processing improved speech perception in noise by a $9 \mathrm{~dB} \mathrm{~S} / \mathrm{N}$ ratio (compared with long electrode subjects). To put this improvement in $\mathrm{S} / \mathrm{N}$ ratio in perspective, Eddington et al. have demonstrated that a $5 \mathrm{~dB}$ improvement in $\mathrm{S} / \mathrm{N}$ ratio is comparable with an approximate $30 \%$ to $40 \%$ improvement in HINT sentence scores. ${ }^{14}$ Although the improvement for speech in noise does not bring the implant patient to normal-hearing levels, it represents a large advance toward that goal. The speech in noise results for this group of severely hearingimpaired individuals using the A + E approach are nearly as good as mild-to-moderate HL patients. Remember that for these individuals, hearing aid technology was unable to improve their speech recognition to acceptable levels in quiet or in noise. It will be interesting to see whether those with more moderate hearing losses can realize similar improvements in noise using the electro-acoustical stimulation paradigm.

Preservation of any residual hearing must be a goal of all future CI surgeries. Implant surgeons should adopt surgical strategies to limit intracochlear damage. The
Fig. 8. The spondee recognition threshold (signal-to-noise $[\mathrm{S} / \mathrm{N}]$ ratio in $\mathrm{dB}$ required for $50 \%$ correct recognition in a multitalker background) for four groups of patients. Error bars represent \pm 1 SD about the mean. Traditional $=$ subjects using standard length electrode; E\&A = subjects using electroacoustical stimulation; mild to moderate loss = subjects using hearing aids; normal $=$ normal hearing subjects.

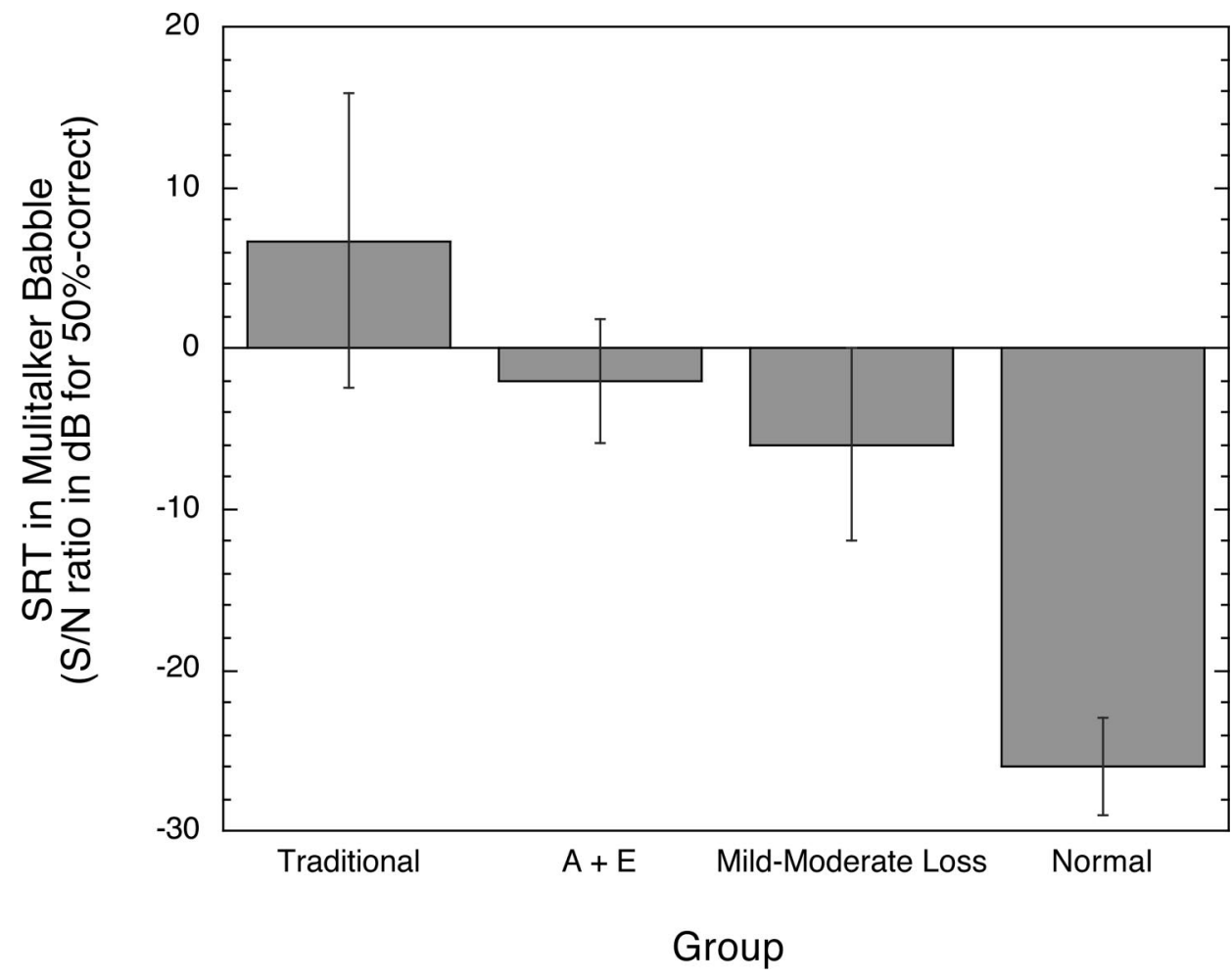

Gantz et al.: Preservation of Hearing in Cochlear Implant Surgery 
ability to enter the inner ear and place a $10 \mathrm{~mm}$ electrode opens many possibilities for future therapies. CI manufactures must recognize the importance of inner ear preservation in all individuals undergoing cochlear implantation and should adjust the design of intracochlear electrodes to accommodate this concept. This research also demonstrates the need to improve low-frequency, fine-spectral perception to CI recipients who do not have residual acoustic hearing to provide more enjoyment of music as well as hearing in noise.

\section{Acknowledgments}

The authors thank the principle investigators who have accrued subjects in the Hybrid Implant clinical trial: Charles Luetje, Kansas City Ear Institute; Noel Cohen, MD, and J. Thomas Roland, MD, New York University; William Luxford, MD, House Ear Institute; Joseph Roberson, MD, California Ear Institute; Peter Roland, MD, University of Texas, Dallas; and Richard Miyamoto, MD, Indiana University.

\section{BIBLIOGRAPHY}

1. Rubinstein JT, Parkinson WS, Tyler RS, Gantz BJ. Residual speech recognition and cochlear implant performance: Effects of implantation criteria. Am J Otol 1999;20:445-452.

2. Fishman K, Shannon RV, Slattery W. Speech recognition as a function of the number of electrodes used in the SPEAK cochlear implant strategy. J Speech Hear Res 1988;40: 1201-1215.

3. Fu Q-J, Shannon RV, Wang X. Effects of noise and spectral resolution on vowel and consonant recognition: Acoustic and electric hearing. $J$ Acoust Soc Am 1998;104: 3586-3596.

4. Friesen L, Shannon RV, Baskent D, Wang X. Speech recognition in noise as a function of the number of spectral channels: Comparison of acoustic hearing and cochlear implants. J Acoust Soc Am 2001;110:1150-1163.

5. Turner CW, Gantz BJ, Vidal C, et al A. Speech recognition in noise for cochlear implant listeners: benefits of residual acoustic hearing. J Acoust Soc Am 2004;115:1729-1735.

6. Stickney GS, Zeng FG, Litovsky RV, Assman PF. Cochlear implant speech recognition with speech maskers. J Acoust Soc Am 2004;116:1081-1091.

7. Gfeller KE, Turner C, Woodworth G, et al. Recognition of familiar melodies by adult cochlear implant recipients and normal hearing adults. Cochlear Implants Int 2002;3: $29-53$.

8. Gfeller K, Lansing C. Melodic, rhythmic, and timbral perception of adult cochlear implant users. J Speech Hear Res 1991;34:916-920.

9. Kong Y, Cruz R, Jones J, Zeng F. Music perception with temporal cues in acoustic and electric hearing. Ear Hear 2004;25:173-185.

10. Gantz BJ, Turner CW. Combining Acoustic and Electric Hearing. Laryngoscope 2003;113:1726-1730.

11. Gantz BJ, Turner CW. Combining acoustic and electrical speech processing: Iowa/Nucleus hybrid implant. Acta Otolaryngol 2004;124:344-347.

12. Greenwood DD. A cochlear frequency position function for several species: 29 years later. J Acoust Soc Am 1990;87: 2592-2605.

13. Peterson FE, Lehiste I. Revised CNC lists for auditory tests. J Speech Hear Dis 1962;27:62-70.

14. Eddington DK, Rabinowitz WR, Tierney J, et al. Speech processors for auditory prostheses. In: 8th Quarterly Progress Report NIDCD Contract N01-6-2100; 1997. 